\title{
Anti-inflammatory effects on murine macrophages of ethanol extracts of Lygodium japonicum spores via inhibition of NF-kB and p38
}

\author{
YOUNG-CHANG CHO, BA REUM KIM, HIEN THI THU LE and SAYEON CHO \\ Laboratory of Molecular Pharmacological Cell Biology, College of Pharmacy, \\ Chung-Ang University, Seoul 06974, Republic of Korea
}

Received February 24, 2017; Accepted June 28, 2017

DOI: $10.3892 / \mathrm{mmr} .2017 .7070$

\begin{abstract}
The spores of Lygodium japonicum (Thunb.) Sw. (L.japonicum) have been used in traditional Chinese medicine for the treatment of various inflammatory diseases. However, the molecular mechanisms underlying their anti-inflammatory effects have yet to be elucidated. In the present study, we investigated the anti-inflammatory effects of ethanol extracts of L. japonicum spores (ELJ) by measuring the production of inflammatory mediators, and explored the molecular mechanisms underlying the effects of ELJ in murine macrophages in vitro using immunoblotting analyses. At non-cytotoxic concentrations of $(50-300 \mu \mathrm{g} / \mathrm{ml})$, ELJ was revealed to significantly suppress the production of nitric oxide (NO) and tumor necrosis factor (TNF)- $\alpha$ in lipopolysaccharide (LPS)-stimulated murine RAW 264.7 macrophages; ELJ repressed the production of interleukin (IL)- 6 only at high concentrations ( $\geq 200 \mu \mathrm{g} / \mathrm{ml})$. The ELJ-mediated decrease in NO production was demonstrated to depend on the downregulation of inducible NO synthase mRNA and protein expression. Conversely, the mRNA and protein expression of cyclooxygenase- 2 were not affected by ELJ. In addition, ELJ was revealed to inhibit the mRNA expression of IL-6, IL-1 $\beta$, and TNF- $\alpha$ in LPS-stimulated RAW 264.7 macrophages. The effects of ELJ on proinflammatory mediators may have been due to the stabilization of inhibitor of $\kappa \mathrm{B} \alpha$ and the inhibition of p38 mitogen-activated protein kinase (MAPK). These results suggested that ELJ may suppress LPS-induced inflammatory responses in murine macrophages in vitro, through the negative regulation of $\mathrm{p} 38 \mathrm{MAPK}$ and nuclear factor (NF)- $\mathrm{\kappa B}$. Therefore, ELJ may have potential as a novel candidate for
\end{abstract}

Correspondence to: Professor Sayeon Cho, Laboratory of Molecular and Pharmacological Cell Biology, College of Pharmacy, Chung-Ang University, 84 Heukseok-Ro, Dongjak-Gu, Seoul 06974, Republic of Korea

E-mail: sycho@cau.ac.kr

Key words: Lygodium japonicum spores, macrophages, lipopolysaccharide, inflammatory mediators, nuclear factor- $\mathrm{\kappa B}$, p38 the development of therapeutic strategies aimed at alleviating inflammation.

\section{Introduction}

Inflammation is an important immune response that serves to protect the host against pathogen infections or tissue injury. During inflammatory responses, macrophages are the major immune cells that regulate innate or adaptive immunity (1). Macrophages are activated by infection and tissue injury, and produce proinflammatory mediators, including interleukin (IL)-1 $\beta$, IL-6, tumor necrosis factor (TNF)- $\alpha$, nitric oxide (NO), and prostaglandin ( $\mathrm{PG}) \mathrm{E}_{2}$ (2); proinflammatory mediators then induce inflammatory responses in adjacent tissues and activate neighboring immune cells (3). The production of inflammatory mediators is regulated through intracellular signaling pathways, including mitogen-activated protein kinase (MAPK) and nuclear factor (NF)- $\mathrm{kB}$ pathways $(4,5)$. However, the aberrant activation of these pathways, as a result of persistent infection and continuous exposure to chemical stimuli, may lead to chronic inflammation (6,7). Chronic inflammation is induced and maintained by increased levels of proinflammatory mediators and can result in several inflammatory diseases, including rheumatoid arthritis, inflammatory bowel disease, asthma, Alzheimer's disease and chronic obstructive pulmonary disease (8-10).

The spores of Lygodium japonicum (Thunb.) Sw. (L. japonicum) are also called Lygodii Spora, and are widely distributed in East Asia and Australia. The L.japonicum plant, its spores or its roots have been used in traditional medicine in several Asian countries, including China, Korea, India, Nepal and Pakistan, for the treatment of various diseases, including inflammatory disorders, swelling, ulcers, Herpes infections, wounds, dysuria, skin eczema, nephritis, enteritis, diarrhea and urinary tract infections (11-14). Ethanol extracts of L. japonicum spores (ELJ) have been reported to inhibit testosterone $5 \alpha$-reductase and may have potential for the treatment of benign prostatic hyperplasia (13). In addition, ELJ has been revealed to significantly reduce chronic inflammation in ethylene glycol-induced kidney calculi rat models (15), while methanol extracts have demonstrated neuroprotective effects against glutamate-induced neurotoxicity (16). Several active 
components, including fatty acids, flavones, phenolic acids and phenolic glycosides, have been identified in L. japonicum spores and may be responsible for its effects (17-19).

Despite its widespread traditional use, the molecular mechanisms underlying the anti-inflammatory effects of L. japonicum have yet to be elucidated. In the present study, the anti-inflammatory effects of ELJ on the production of proinflammatory mediators were investigated in lipopolysaccharide (LPS)-stimulated murine macrophage cells, and the roles of inflammatory signal transduction pathways in the mechanisms underlying the effects of ELJ were explored in vitro.

\section{Materials and methods}

Cell culture. The murine RAW 264.7 macrophage cell line and the 293 cell line (American Type Culture Collection, Manassas, VA, USA) were cultured in Dulbecco's modified Eagle's medium (DMEM; GE Healthcare Life Sciences, Little Chalfont, UK) supplemented with $10 \%$ fetal bovine serum (FBS; GE Healthcare Life Sciences), $50 \mathrm{U} / \mathrm{ml}$ penicillin and $50 \mu \mathrm{g} / \mathrm{ml}$ streptomycin (Gibco; Thermo Fisher Scientific, Inc., Waltham, MA, USA) throughout the study. Cells were maintained and treated at $37^{\circ} \mathrm{C}$ in a humidified $5 \% \mathrm{CO}_{2}$ atmosphere.

Reagents. A 95\% ethanol extract of L. japonicum spores (cat no. PBC417A) was obtained from Korea Plant Extract Bank (KPEB, Daejeon, Korea). A stock solution $(200 \mathrm{mg} / \mathrm{ml})$ of the extract was prepared in dimethyl sulfoxide (DMSO; Sigma-Aldrich; Merck KGaA, Darmstadt, Germany) and stored at $-20^{\circ} \mathrm{C}$ until use. Rabbit polyclonal anti-inhibitor of $\kappa \mathrm{B} \alpha(\mathrm{I} \kappa \mathrm{B} \alpha$; cat no. sc-371), anti-IкB kinase (IKK) $\alpha / \beta$ (cat no. sc-7607) and mouse monoclonal anti- $\alpha$-tubulin (cat no. sc-5286) antibodies were purchased from Santa Cruz Biotechnology, Inc. (Dallas, TX, USA). Rabbit polyclonal anti-inducible NO synthase (iNOS; cat no. 2982), anti-cyclooxygenase (COX)-2 (cat no. 4842), monoclonal anti-phosphorylated (p)-IкB $\alpha$ (Ser32/36; cat no. 9246), polyclonal anti-p38 MAPK (cat no. 9212), anti-p-p38 (Thr180/Tyr182; cat no. 9211), anti-extracellular signal-regulated kinase (ERK) 1/2 (cat no. 9102), anti-c-Jun N-terminal kinase (JNK; cat no. 9252), anti-p-JNK (Thr183/Tyr185; cat no. 9251), monoclonal anti-p-IKK $\alpha / \beta$ (Ser176/180; cat no. 2697), polyclonal anti-p-MAPK kinase (MKK) 3/6 (Ser189/207; cat no. 9231), anti-MKK6 (cat no. 9264), anti-transforming growth factor $\beta$-activated kinase 1 (TAK1; cat no. 4505), monoclonal anti-p-TAK1 (Thr184/187; cat no. 4508) and mouse monoclonal anti-p-ERK1/2 (Thr202/Tyr204; cat no. 9106) were purchased from Cell Signaling Technology, Inc. (Danvers, MA, USA). EZ-Cytox Cell Viability, Proliferation \& Cytotoxicity Assay kit was purchased from Daeil Lab Service, Co., Ltd. (Seoul, Korea). Ready-SET-Go! ELISA kits for the detection of IL-6 (cat no. 88-7064) and TNF- $\alpha$ (cat no. 88-7324) were obtained from eBioscience (Thermo Fisher Scientific, Inc.). Accuzol Total RNA Extraction solution was from Bioneer Corporation (Daejeon, Korea) and TOPscript cDNA Synthesis kit was from Enzynomics, Co., Ltd. (Daejeon, Korea). iTaq Universal SYBR Green Supermix was from Bio-Rad Laboratories, Inc. (Hercules, CA, USA).
NO production assay. RAW 264.7 macrophages were seeded in 96 -well plates at a density of $4.0 \times 10^{4}$ cells/well and incubated at $37^{\circ} \mathrm{C}$ overnight. Subsequently, cells were pre-treated with various concentrations of ELJ $(50,100,200$ and $300 \mu \mathrm{g} / \mathrm{ml})$ or vehicle (DMSO) at $37^{\circ} \mathrm{C}$ for $2 \mathrm{~h}$ prior to stimulation with LPS (Sigma-Aldrich; Merck KGaA) at $37^{\circ} \mathrm{C}$. Following stimulation with LPS $(1 \mu \mathrm{g} / \mathrm{ml})$ for $24 \mathrm{~h}$, the supernatants were collected by centrifugation at $1,500 \mathrm{x}$ g for $1 \mathrm{~min}$ at room temperature and transferred to 96-well plates. After that, $100 \mu \mathrm{l}$ Griess reagent ( $1 \%$ sulfanilamide, $0.1 \% \mathrm{~N}$-1-naphthylenediamine dihydrochloride and $2.5 \%$ phosphoric acid) was added to each well at room temperature for $1 \mathrm{~min} . \mathrm{NaNO}_{2}$ solutions $(2.5,5,10,25$, 50 and $100 \mu \mathrm{M}$ ) were used to construct a standard curve based on the least squares method to calculate the quantity of NO in the supernatant samples. The absorbance of each sample was measured at $540 \mathrm{~nm}$ using a Synergy H1 Hybrid Microplate Reader (BioTek Instruments, Inc., Winooski, VT, USA).

Cell viability assay. RAW 264.7 macrophages were pre-treated with $\operatorname{ELJ}(50,100,200,300$ and $400 \mu \mathrm{g} / \mathrm{ml})$ for $2 \mathrm{~h}$, followed by an additional $24 \mathrm{~h}$ of incubation at $37^{\circ} \mathrm{C}$ in the absence or presence of LPS (1 $\mu \mathrm{g} / \mathrm{ml})$. Subsequently, $25 \mu \mathrm{l}$ EZ-Cytox solution was added to each well in $250 \mu \mathrm{l}$ medium and cells were incubated for $1 \mathrm{~h}$ at $37^{\circ} \mathrm{C}$. Supernatants were transferred to new 96 -well plates and cell viability was measured by calculating the absorbance at 450 and $650 \mathrm{~nm}\left(\mathrm{~A}_{450}-\mathrm{A}_{650}\right)$ using Synergy H1 Hybrid Microplate Reader.

Enzyme-linked immunosorbent assay (ELISA). RAW 264.7 macrophages were treated with various concentrations ELJ (50, 100, 200 and $300 \mu \mathrm{g} / \mathrm{ml}$ ) or vehicle (DMSO) for $2 \mathrm{~h}$, followed by stimulation with LPS $(1 \mu \mathrm{g} / \mathrm{ml})$ for $24 \mathrm{~h}$. Subsequently, the supernatants were collected after centrifugation at $1,500 \mathrm{x} g$ for $1 \mathrm{~min}$ at room temperature and diluted according to the predetermined dilution ratio for each proinflammatory cytokine. The production of the proinflammatory cytokines IL-6 and TNF- $\alpha$ was measured using commercially available ELISA kits according to the manufacturer's protocol. Briefly, a 96-well plate was coated with the coating solution at $4^{\circ} \mathrm{C}$ overnight, washed 3 times with $1 \mathrm{X}$ PBS containing $0.05 \%$ Tween-20 (PBST), and treated with 1X Assay Diluent for $1 \mathrm{~h}$ at room temperature. The solution was removed, and the supernatants (diluted as appropriate) and standard solutions were added to the wells. Following $2 \mathrm{~h}$ of treatment at room temperature, the plate was washed 3 times with 1X PBST and the detection antibody diluted in 1X Assay Diluent was added to the plate for $1 \mathrm{~h}$ at room temperature. The plate was then washed with $1 \mathrm{X}$ PBST, and 1X horseradish peroxidase (HRP)-streptavidin solution diluted in 1X Assay Diluent was added for $30 \mathrm{~min}$ at room temperature, followed by washing 5 times with $1 \mathrm{X}$ PBST. The 3,3',5,5'-tetramethylbenzidine solution was then added to the plate and incubated for $10 \mathrm{~min}$ at room temperature in the dark. Subsequently, $1 \mathrm{~N} \mathrm{H}_{3} \mathrm{PO}_{4}$ was added to the plate to stop the reaction, and the absorbance of each well was measured using the Synergy H1 Hybrid Microplate Reader at $450 \mathrm{~nm}$.

RNA preparation and cDNA synthesis. RAW 264.7 macrophages were seeded into a 12 -well plate at a density of $8 \times 10^{5}$ cells/well and incubated at $37^{\circ} \mathrm{C}$ overnight. Cells were 
pre-treated with $\operatorname{ELJ}(50,100,200$ and $300 \mu \mathrm{g} / \mathrm{ml})$ for $2 \mathrm{~h}$ and then incubated with LPS $(1 \mu \mathrm{g} / \mathrm{ml})$ for $3 \mathrm{~h}$. Total RNA was extracted from cells using Accuzol Total RNA Extraction solution and was reverse transcribed into cDNA for $1 \mathrm{~h}$ at $37^{\circ} \mathrm{C}$ using the TOPscript cDNA Synthesis kit, according to the manufacturer's protocol.

Quantitative polymerase chain reaction ( $q P C R)$. PCR amplification of cDNA was performed using iTaq Universal SYBR Green Supermix according to the manufacturer's protocol. Thermocycling conditions were as follows: Initial denaturation at $94^{\circ} \mathrm{C}$ for $3 \mathrm{~min}$, followed by 40 cycles of denaturation at $94^{\circ} \mathrm{C}$ for $5 \mathrm{sec}$ and annealing/extension at $60^{\circ} \mathrm{C}$ for $30 \mathrm{sec}$ using a CFX Connect Real-Time PCR Detection system (Bio-Rad Laboratories, Inc.). Using the $2^{-\Delta \Delta C q}$ method (20), gene expression was quantified and normalized to the reference genes $\beta$-actin and GAPDH; gene expression was expressed as a ratio over the expression in the LPS treated group (defined as $100 \%$ ). The primers used for PCR are as follows, in accordance with our previous study (21): iNOS, sense 5'-TGG CCA CCA AGC TGA ACT-3', antisense 5'-TCA TGA TAA CGT TTC TGG CTC TT-3'; COX-2, sense 5'-GAT GCT CTT CCG AGC TGT G-3', antisense 5'-GGA TTG GAA CAG CAA GGA TTT-3'; TNF- $\alpha$, sense 5'-CTG TAG CCC ACG TCG TAG C-3', antisense 5'-TTG AGA TCC ATG CCG TTG-3'; IL-6, sense 5'-TCT AAT TCA TAT CTT CAA CCA AGA GG-3', antisense 5'-TGG TCC TTA GCC ACT CCT TC-3'; IL-1 $\beta$, sense 5'-TTG ACG GAC CCC AAA AGA T-3', antisense 5'-GAT GTG CTG CTG CGA GAT T-3'; $\beta$-actin, sense 5'-CGT CAT ACT CCT GCT TGC TG-3', antisense 5'-CCA GAT CAT TGC TCC TCC TGA-3'; and GAPDH, sense 5'-GCT CTC TGC TCC TCC TGT TC-3' and antisense 5'-ACG ACC AAA TCC GTT GAC TC-3'.

Luciferase reporter assay. HEK 293 cells were seeded into a $100-\mathrm{mm}$ dish. When $70 \%$ confluent, they were transfected using polyethylenimine (Polysciences, Inc., Warrington, PA, USA) as the transfection reagent for $6 \mathrm{~h}$ at $37^{\circ} \mathrm{C}$ with $4.5 \mu \mathrm{g}$ pNF- $\kappa \mathrm{B}-$ luc or pAP-1-luc cis-reporter plasmids (Agilent Technologies, Inc., Santa Clara, CA, USA), which contained the $\mathrm{NF}-\kappa \mathrm{B}$ or activator protein 1 (AP-1) promoter, respectively and the luciferase reporter gene. The gWIZ-green fluorescent protein (GFP) plasmid was used as an internal control for transfection efficiency. Transfected cells were seeded into 12-well plates, incubated overnight at $37^{\circ} \mathrm{C}$, and treated with various concentrations of $\operatorname{ELJ}(50,100,200$ and $300 \mu \mathrm{g} / \mathrm{ml})$ in the presence of phorbol 12-myristate 13-acetate (PMA), as an activator of NF- $\kappa \mathrm{B}$. Following incubation for $24 \mathrm{~h}$, cells were lysed with Cell Culture Lysis Reagent (Promega Corporation, Madison, WI, USA). For determination of luciferase activity, the Luciferase Assay System (cat no. E1500; Promega Corporation) was used. GFP expression was determined by measuring the fluorescence at $525 \mathrm{~nm}$ following excitation at $485 \mathrm{~nm}$ and was used as a control for luciferase activity. The luminescence and fluorescence were measured using Synergy H1 Hybrid Microplate Reader and analyzed using Gen5 software version 1.11.5 (BioTek Instruments, Inc.).

Preparation of total cell lysates. RAW 264.7 macrophages were seeded into a 6 -well plate $\left(2 \times 10^{6}\right.$ cells/well). Then, cells were pre-treated with ELJ $(50,100,200$ and $300 \mu \mathrm{g} / \mathrm{ml})$ for $2 \mathrm{~h}$ at $37^{\circ} \mathrm{C}$ and stimulated with LPS $(1 \mu \mathrm{g} / \mathrm{ml})$ for the detection of the target proteins: For I $\mathrm{IB} \alpha$ and TAK 1 for $3 \mathrm{~min}$; for MAPKs for $15 \mathrm{~min}$; and for iNOS and COX-2 for $24 \mathrm{~h}$. Following stimulation for the indicated times, cells were washed 3 times with ice-cold PBS and lysed with lysis buffer, containing $0.5 \%$ octylphenoxypolyethoxyethanol, $0.5 \%$ Triton $\mathrm{X}-100,150 \mathrm{mM} \mathrm{NaCl}, 20 \mathrm{mM}$ Tris- $\mathrm{HCl}$ (pH 8.0), $1 \mathrm{mM}$ EDTA, $1 \%$ glycerol, $1 \mathrm{mM}$ phenylmethylsulfonyl fluoride, $10 \mathrm{mM} \mathrm{NaF}$ and $1 \mathrm{mM} \mathrm{Na}_{3} \mathrm{VO}_{4}$, for $10 \mathrm{~min}$ at $4^{\circ} \mathrm{C}$. Following centrifugation at $15,814 \mathrm{x} \mathrm{g}$ for $30 \mathrm{~min}$ at $4^{\circ} \mathrm{C}$, the supernatants were collected.

Western blot analysis. Total protein was quantified using a Bradford protein assay, and the mixture of lysates and $5 \mathrm{X}$ Laemmli sample buffer (Bio-Rad Laboratories, Inc.) was boiled for $5 \mathrm{~min}$ at $100^{\circ} \mathrm{C}$. Then, equal amounts of extracted protein samples $(20 \mu \mathrm{g})$ were separated by $10 \%$ SDS-PAGE and transferred onto nitrocellulose membranes using transfer buffer (192 mM glycine, $25 \mathrm{mM}$ Tris- $\mathrm{HCl} \mathrm{pH} 8.8$ and $20 \%$ $\mathrm{v} / \mathrm{v}$ methanol). Membranes were blocked for $1 \mathrm{~h}$ at room temperature with $5 \%$ non-fat dried milk, followed by incubation overnight at $4^{\circ} \mathrm{C}$ with the following primary antibodies (1:1,000 dilution): anti-I $\mathrm{B} \alpha$, anti-IKK $\alpha / \beta$, anti- $\alpha$-tubulin, anti-iNOS, anti-COX-2, anti-p-IкB $\alpha$ (Ser32/36), anti-p38 MAPK, anti-p-p38 (Thr180/Tyr182), anti-ERK1/2, anti-JNK, anti-p-JNK (Thr183/Tyr185), anti-p-IKK $\alpha / \beta$ (Ser176/180), anti-p-MAPK kinase (MKK) 3/6 (Ser189/207), anti-MKK6, anti-TAK, anti-p-TAK1 (Thr184/187) and anti-p-ERK1/2 (Thr202/Tyr204). Subsequently, membranes were incubated for an additional $1 \mathrm{~h}$ at room temperature with HRP-conjugated goat anti-mouse (1:5,000; cat no. LF-SA8001A; Abfrontier; Young In Frontier Co., Ltd., Seoul, Korea) or goat anti-rabbit (1:5,000; cat no. LF-SA8002A; Abfrontier; Young In Frontier Co., Ltd.) secondary antibodies. After washing 5 times with $1 \mathrm{X}$ PBS containing $0.5 \%$ Tween-20, the immunoreactive bands were visualized using enhanced chemiluminescence. Blots were semi-quantified by densitometry using VisionWorksLS Analysis software (UVP, LLC, Upland, CA, USA).

Statistical analysis. Data are presented as the mean \pm standard error of the mean of 3 independent experiments with 3 replicates each. The statistical significance of the differences between groups was assessed using one-way analysis of variance followed by a post hoc Dunnett's test for multiple comparisons. Statistical analysis was performed using GraphPad Prism software version 3.0 (GraphPad Software, Inc., La Jolla, CA, USA). P<0.05 was considered to indicate a statistically significant difference.

\section{Results}

Effects of ELJ treatment on cell viability. Since the cell-based evaluation of potential anti-inflammatory agents should be performed under non-cytotoxic conditions, the maximal non-cytotoxic concentration of ELJ was initially determined in murine RAW 264.7 macrophages. Macrophages were treated with various concentrations of ELJ in the presence or absence of stimulation with LPS. Cell viability was determined based on the ability of cells to metabolically reduce a tetrazolium salt 

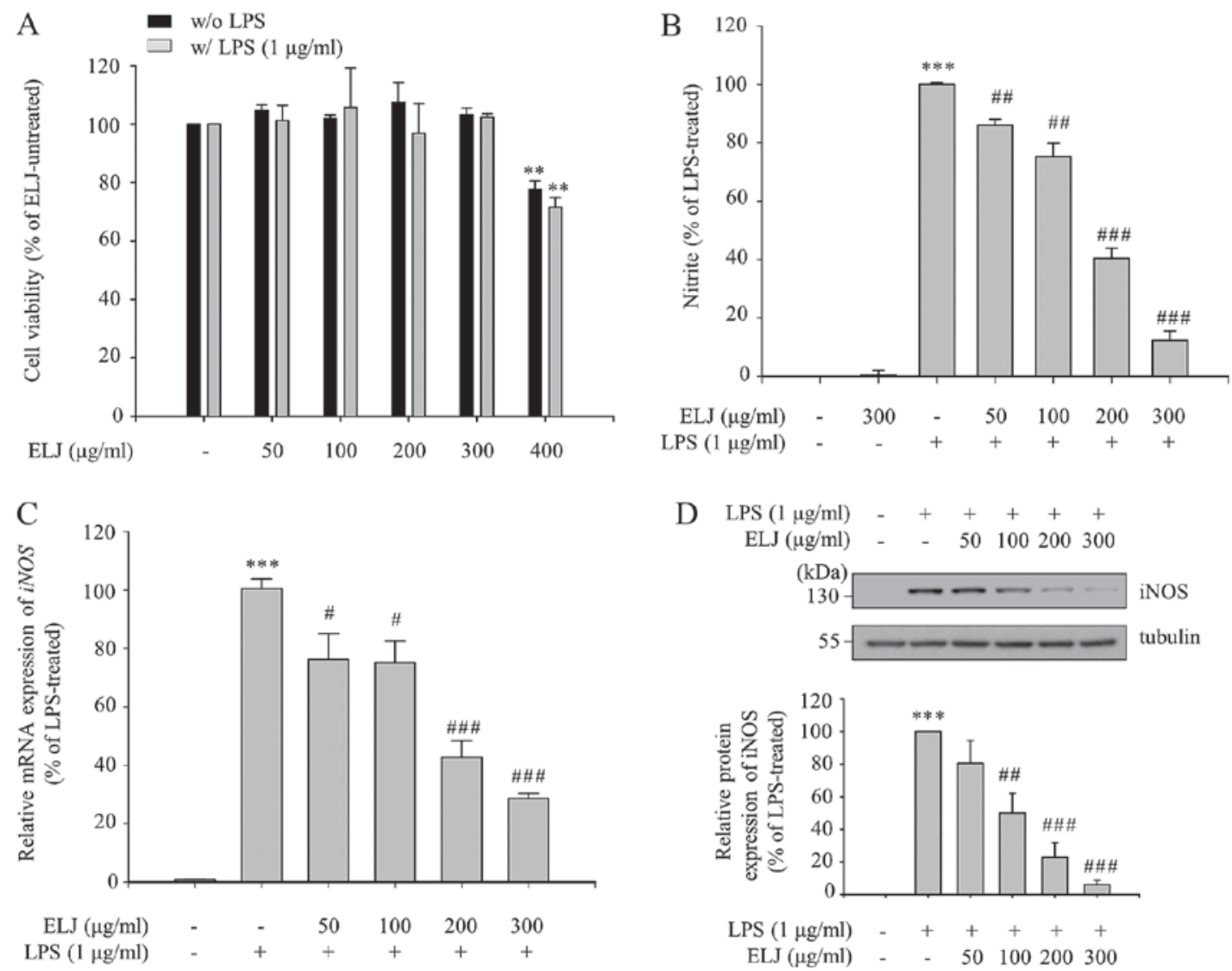

Figure 1. Effects of ELJ on cell viability and NO production. RAW 264.7 macrophages were pre-treated with ELJ, followed by incubation in the presence or absence of LPS. (A) After incubation for $24 \mathrm{~h}$, cell viability was determined. Data are presented as the mean $\pm \mathrm{SEM}$. ** $\mathrm{P}<0.01$ vs. ELJ-treated control groups with or without LPS stimulation. (B) After incubation for $24 \mathrm{~h}$, NO levels in the culture supernatants were measured using Griess reagent. Data are presented the mean \pm SEM. ${ }^{* * *} \mathrm{P}<0.001$ vs. LPS-untreated control groups; ${ }^{\# \#} \mathrm{P}<0.01$ and ${ }^{\# \# \#} \mathrm{P}<0.001$ vs. LPS-treated groups. (C) After incubation for $3 \mathrm{~h}$, total RNA was extracted and iNOS mRNA expression levels were assessed using reverse transcription-quantitative polymerase chain reaction. Data are presented as the mean \pm SEM. ${ }^{* * *} \mathrm{P}<0.001$ vs. LPS-untreated control groups; ${ }^{~} \mathrm{P}<0.05$ and ${ }^{\# \# \#} \mathrm{P}<0.001$ vs. LPS-treated groups. (D) Total cell lysates were prepared after $24 \mathrm{~h}$ of incubation and the protein expression of iNOS was detected by immunoblotting. Data are presented as the mean \pm SEM. ${ }^{* * *} \mathrm{P}<0.001$ vs. LPS-untreated control groups; ${ }^{\# \#} \mathrm{P}<0.01$ and ${ }^{\# \#} \mathrm{P}<0.001$ vs. LPS-treated groups. ELJ, ethanol extract of Lygodium japonicum spores; NO, nitric oxide; LPS, lipopolysaccharide; SEM, standard error of the mean; iNOS, inducible NO synthase.

to a formazan dye: As presented in Fig. 1A, ELJ did not exert cytotoxic effects at concentrations $<300 \mu \mathrm{g} / \mathrm{ml}$, regardless of LPS stimulation. However, cell viability was significantly reduced at a high concentration of ELJ (400 $\mu \mathrm{g} / \mathrm{ml}$; Fig. 1A). Therefore, ELJ concentrations $<300 \mu \mathrm{g} / \mathrm{ml}$ were selected for subsequent experiments.

ELJ inhibits LPS-induced iNOS expression and NO production. Since NO serves a key role in inflammatory responses (22), the present study investigated the effects of ELJ on LPS-induced NO production. The levels of NO in RAW 264.7 macrophages were significantly decreased following treatment with ELJ in a dose-dependent manner (Fig. 1B). In addition, the mRNA and protein expression levels of iNOS in ELJ-treated macrophages were analyzed using RT-qPCR and western blot analysis, respectively, since iNOS is a key regulator of NO production during inflammatory responses (23). As presented in Fig. 1C and D, treatment with ELJ resulted in a dose-dependent downregulation in iNOS mRNA and protein expression levels in LPS-stimulated macrophages. These findings suggested that ELJ may counteract the LPS-induced increase in NO production through suppressing iNOS expression in macrophages in vitro.
ELJ suppresses the LPS-induced production of proinflammatory mediators. COX-2 is a proinflammatory enzyme that regulates the production of $\mathrm{PGE}_{2}$ (24). RT-qPCR and western blot analysis revealed that treatment with ELJ did not affect the mRNA and protein expression levels of COX-2 in LPS-stimulated macrophages (Fig. 2A and B). These results suggested that ELJ may selectively suppress the expression of iNOS but not COX-2 in macrophages in vitro.

Proinflammatory cytokines, including IL-1 $\beta$, IL-6 and TNF- $\alpha$, are induced following LPS stimulation and serve pivotal roles during LPS-mediated inflammatory responses (3). Therefore, the effects of ELJ on the expression of proinflammatory cytokines were investigated at the mRNA and protein level. As presented in Fig. $2 \mathrm{C}$, the production of TNF- $\alpha$ was significantly inhibited by ELJ in LPS-stimulated RAW 264.7 macrophages in a dose-dependent manner. In addition, treatment with high concentrations of ELJ (200-300 $\mu \mathrm{g} / \mathrm{ml}$ ) was revealed to suppress the production of IL-6 (Fig. 2D). Furthermore, RT-qPCR demonstrated that ELJ downregulated the mRNA expression of proinflammatory cytokines (Fig. 2E). These findings suggested that ELJ may exert anti-inflammatory effects, via inhibiting the production of proinflammatory cytokines in vitro. 


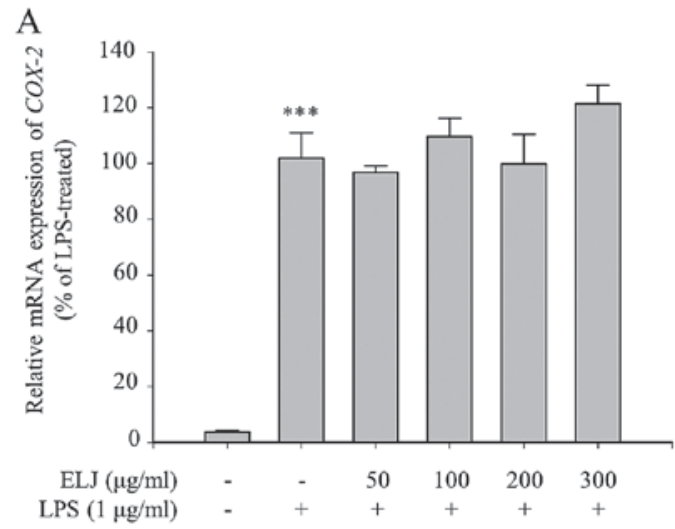

B
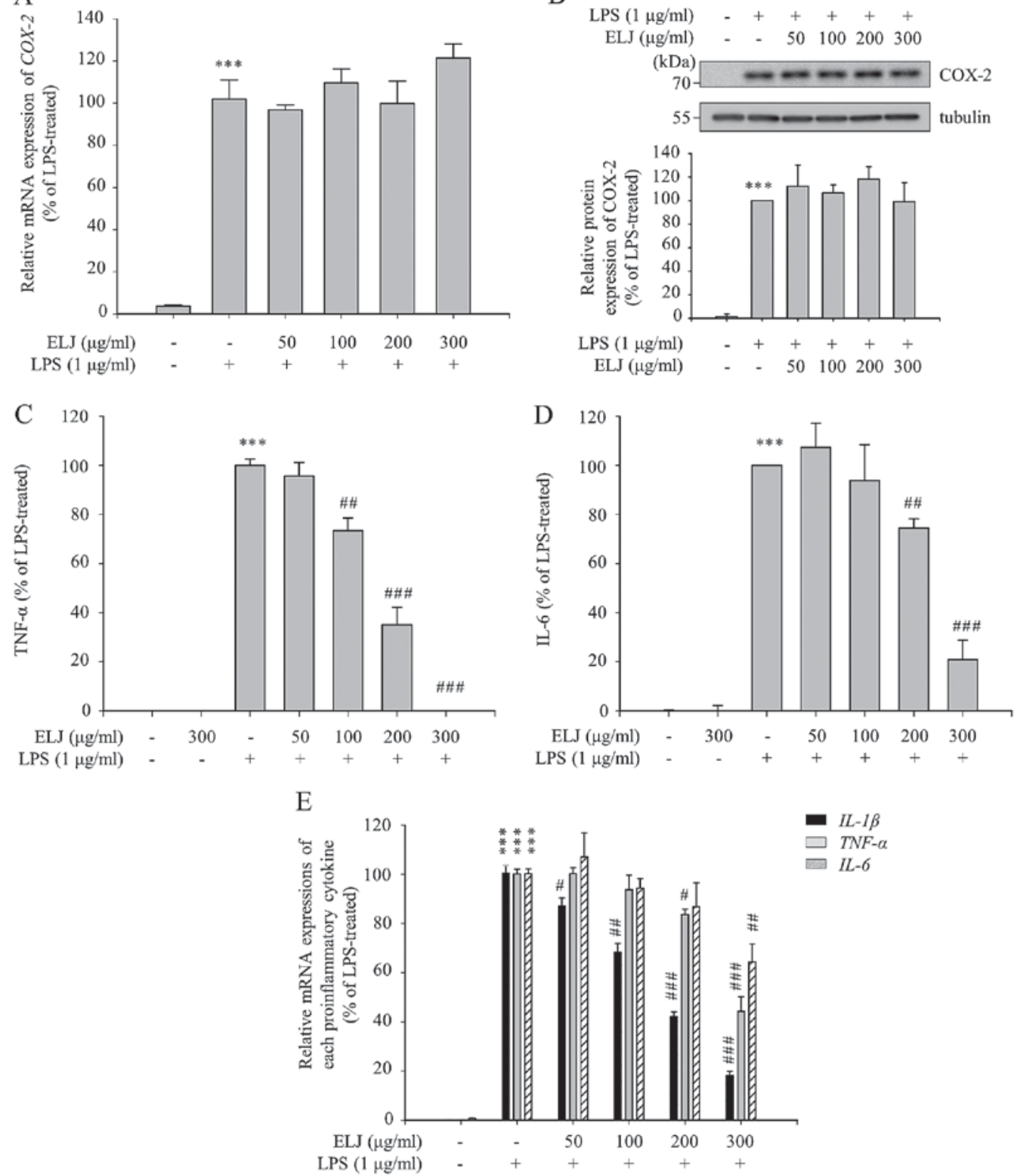

Figure 2. Effects of ELJ on the expression of COX-2 and the production of proinflammatory cytokines. RAW 264.7 macrophages were pre-treated with ELJ, followed by incubation with LPS. (A) After incubation for $3 \mathrm{~h}$, total RNA was extracted and COX-2 expression levels were assessed using RT-qPCR. Data are presented as the mean \pm SEM. ${ }^{* * *} \mathrm{P}<0.001$ vs. LPS-untreated control groups. (B) Total cell lysates were prepared after $24 \mathrm{~h}$ of incubation and the protein expression of COX-2 was by immunoblotting. Data are presented as the mean \pm SEM. ${ }^{* * *} \mathrm{P}<0.001$ vs. LPS-untreated control groups. (C and D) After incubation for $24 \mathrm{~h}$, TNF- $\alpha$ and IL-6 levels in the culture supernatants were measured using ELISA. (C) TNF- $\alpha$ and (D) IL-6 levels are presented as the mean \pm SEM. ${ }^{* * * *} \mathrm{P}<0.001$ vs. LPS-untreated control groups; ${ }^{\# \#} \mathrm{P}<0.01$ and ${ }^{\# \# \#} \mathrm{P}<0.001$ vs. LPS-treated groups. (E) After incubation for $3 \mathrm{~h}$, total RNA was extracted and IL-6, IL-1 $\beta$ and TNF- $\alpha$ expression levels were assessed using RT-qPCR. Data are presented as the mean \pm SEM. ${ }^{* * *} \mathrm{P}<0.001$ vs. LPS-untreated control groups; ${ }^{\#} \mathrm{P}<0.05,{ }^{\# \#} \mathrm{P}<0.01$ and ${ }^{\# \# \#} \mathrm{P}<0.001$ vs. LPS-treated groups. ELJ, ethanol extract of Lygodium japonicum spores; COX, cyclooxygenase; LPS, lipopolysaccharide; RT-qPCR, reverse transcription-quantitative polymerase chain reaction; SE, standard error of the mean; TNF, tumor necrosis factor; IL, interleukin.

ELJ inhibits $N F-\kappa B$ and p38 signaling pathways. To investigate whether the regulation of transcription may be involved in the inhibitory effects of ELJ on proinflammatory mediator production, the transcriptional activity of NF- $\mathrm{NB}$ and AP-1 was assessed in vitro. $\mathrm{NF}-\kappa \mathrm{B}$ and $\mathrm{AP}-1$ are major transcription factors implicated in the inflammatory response; AP-1 is phosphorylated and thus activated by MAPKs (25). A luciferase reporter assay was used in HEK 293 cells treated with PMA, which induces the transcriptional activation of NF- $\kappa \mathrm{B}-$ and AP-1-dependent genes (5); the luciferase reporter gene was placed under the control of $\mathrm{NF}-\kappa \mathrm{B}$ or AP-1 transcriptional activity. As presented in Fig. $3 \mathrm{~A}$ and $\mathrm{B}$, the $\mathrm{NF}-\kappa \mathrm{B}-$ and AP-1-regulated luciferase activity was significantly induced following treatment with PMA. However, luciferase activity was revealed to be suppressed following ELJ treatment in PMA-stimulated cells. These findings suggested that ELJ may exert anti-inflammatory effects through the inhibition of $\mathrm{NF}-\kappa \mathrm{B}$ and AP-1 transcriptional activity.

Since the production of proinflammatory mediators is regulated by $\mathrm{NF}-\kappa \mathrm{B}$ and MAPK signaling pathways in LPS-stimulated macrophages $(4,5)$, the effects of ELJ on $\mathrm{NF}-\kappa \mathrm{B}$ and MAPK signaling were evaluated in the 

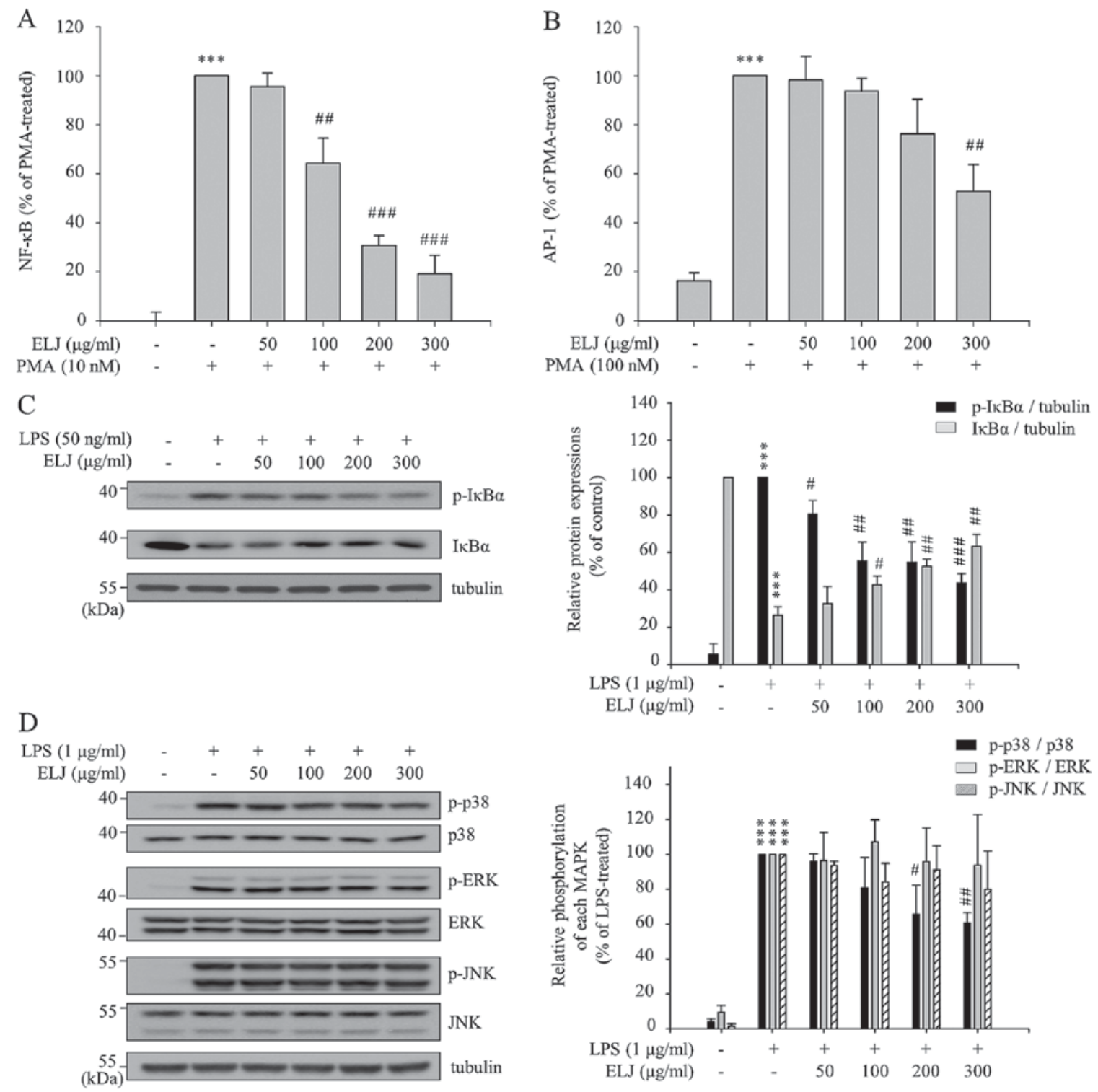

Figure 3. Inhibitory effects of ELJ on the transcriptional activity of NF- $\mathrm{B}$ and AP-1. (A and B) HEK 293 cells were transfected with (A) pNF- $\kappa$ B-luc or (B) pAP-1-luc cis-reporter plasmids. Transfected cells were pre-treated with ELJ, incubated with PMA and luciferase activity was measured. Data are presented as the mean \pm SEM. ${ }^{* * *} \mathrm{P}<0.001$ vs. PMA-untreated control groups; ${ }^{\# \#} \mathrm{P}<0.01$ and ${ }^{\# \# \#} \mathrm{P}<0.001$ vs. PMA-treated groups. (C and D) Total cell lysates

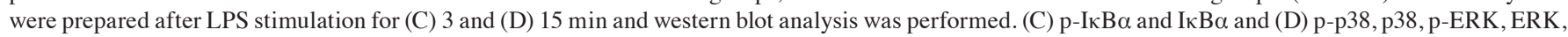
p-JNK and JNK protein expression levels were determined. Data are presented as the mean \pm SEM. ${ }^{* * *} \mathrm{P}<0.001$ vs. LPS-untreated control groups; ${ }^{*} \mathrm{P}<0.05$, ${ }^{\# \#} \mathrm{P}<0.01$ and ${ }^{\# \#} \mathrm{P}<0.001$ vs. LPS-treated groups. ELJ, ethanol extract of Lygodium japonicum spores; NF, nuclear factor; AP, activator protein; HEK, human embryonic kidney; luc, luciferase; PMA, phorbol 12-myristate 13-acetate; SEM, standard error of the mean; LPS, lipopolysaccharide; p-, phosphorylated; IкB, inhibitor of $\kappa \mathrm{B} \alpha$; ERK, extracellular signal-regulated kinase; JNK, c-Jun N-terminal kinase.

present study. I $\kappa \mathrm{B} \alpha$ binds $\mathrm{NF}-\kappa \mathrm{B}$ and stabilizes it in its inactive state, whereas the phosphorylation of $\mathrm{I} \kappa \mathrm{B} \alpha$ at $\mathrm{Ser} 32 / 36$ is the prerequisite for its dissociation from $N F-\kappa B$ and its subsequent recognition by ubiquitin conjugating enzyme E2 D3, leading to $\mathrm{I} \kappa \mathrm{B} \alpha$ polyubiquitination and degradation $(26,27)$. Western blot analysis demonstrated that ELJ suppressed the LPS-induced phosphorylation and degradation of $\mathrm{I} \kappa \mathrm{B} \alpha$ in a dose-dependent manner (Fig. 3C). In addition, the effects of ELJ on the phosphorylation of p38, ERK and JNK, which are major mediators of MAPK signaling, were assessed. MAPK phosphorylation at the phosphorylation sites in their activation loops results in their activation, and the subsequent activation of AP-1, which binds to the promoter of inflammatory mediator genes and induces their transcription $(28,29)$. As presented in
Fig. 3D, treatment with ELJ inhibited the phosphorylation of p38 in LPS-stimulated macrophages in vitro; however, it exerted no effects on the phosphorylation of ERK and JNK, as indicated by western blot analysis. The phosphorylation status of MAPKs is directly related to their kinase activity; therefore, these results suggested that ELJ may suppress the production of proinflammatory mediators via inhibiting NF- $\kappa \mathrm{B}$ and $\mathrm{p} 38$ signaling pathways.

To further investigate the roles of ELJ in the regulation of $\mathrm{NF}-\kappa \mathrm{B}$ and p38 signaling, its effects on the phosphorylation of IKK $\alpha / \beta$ and MKK3/6 were explored; IKK $\alpha / \beta$ and MKK3/6 are upstream kinases of $\mathrm{I} \kappa \mathrm{B} \alpha$ and $\mathrm{p} 38$, respectively $(30,31)$. As demonstrated in Fig. 4A, treatment with ELJ inhibited the LPS-induced phosphorylation of IKK $\alpha / \beta$ and MKK3/6, 

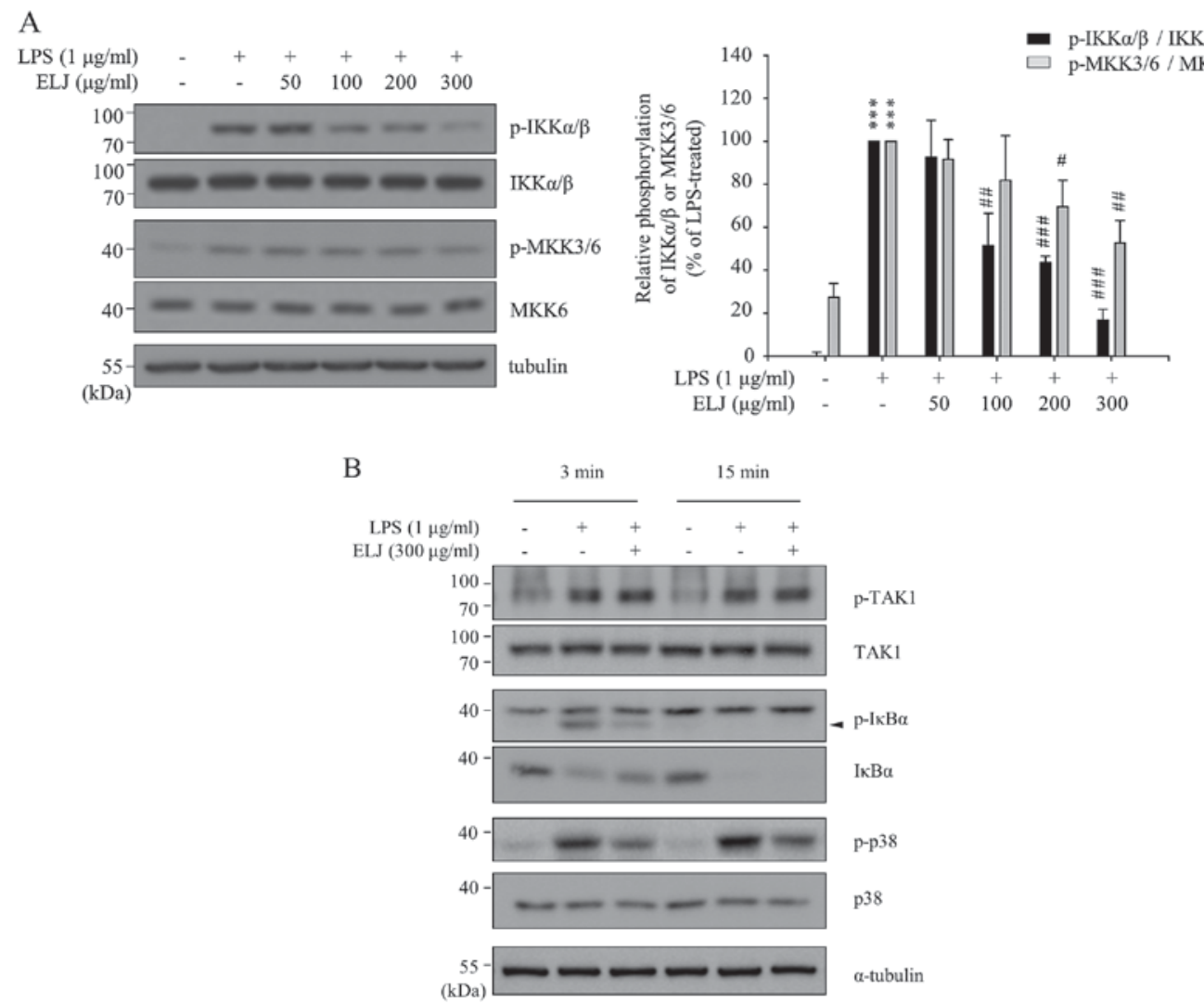

Figure 4. Regulatory effects of ELJ on the phosphorylation of signaling molecules upstream of nuclear factor- $\mathrm{kB}$ and activator protein-1. (A) Total cell lysates from RAW 264.7 macrophages pre-treated with ELJ and incubated with LPS were used for western blot analysis. p-IKK $\alpha / \beta$, IKK $\alpha / \beta$, p-MKK3/6 and MKK6 protein expression levels were detected. Data are presented as the mean $\pm \mathrm{SEM}$. ${ }^{* * *} \mathrm{P}<0.001$ vs. LPS-untreated control groups; ${ }^{*} \mathrm{P}<0.05,{ }^{\# \#} \mathrm{P}<0.01$ and

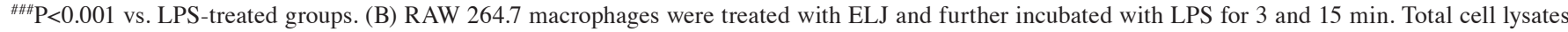
were used for western blot analysis to detect the protein expression levels of TAK1, IкB $\alpha$ and p38. ELJ, ethanol extract of Lygodium japonicum spores; LPS, lipopolysaccharide; p-, phosphorylated; IKK, inhibitor of $\mathrm{\kappa B}$ kinase; MKK, mitogen-activated protein kinase kinase; SEM, standard error of the mean; TAK, transforming growth factor $\beta$-activated kinase; I $\mathrm{I} B \alpha$, inhibitor of $\kappa \mathrm{B} \alpha$.

without affecting the total expression levels of the proteins. However, the LPS-induced phosphorylation of TAK1, which is an upstream kinase of NF- $\mathrm{KB}$ and MAPKs, did not appear to be affected following ELJ treatment (Fig. 4B). These findings suggested that ELJ may target factors located downstream of TAK1 and upstream of IKK $\alpha / \beta$ and MKK3/6 in the regulation of NF- $\kappa \mathrm{B}$ and p38 signaling (Fig. 5).

\section{Discussion}

During inflammation, iNOS is activated and catalyzes the synthesis of NO, which is a free radical synthesized from L-arginine that can cause cellular damage at sites of inflammation (32). In addition, activated macrophages produce proinflammatory cytokines, including TNF- $\alpha$, IL-1 $\beta$ and IL-6 (33). Proinflammatory cytokines stimulate an increase in blood flow and capillary permeability, which can lead to immune cell infiltration, and further potentiate the inflammatory response (33). However, the aberrant excessive production of iNOS and proinflammatory cytokines, as a result of external stimuli, has been associated with the pathophysiology of severe inflammatory disorders, including sepsis and arthritis (34). Previous studies have reported that natural products which inhibit the production of proinflammatory mediators may have potential as phytomedicines for

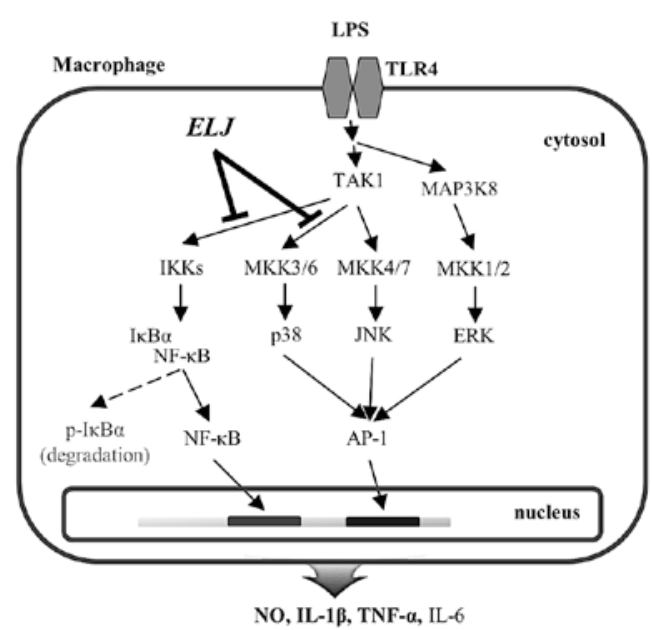

Figure 5. The putative molecular pathway underlying the regulatory effects of ELJ on inflammatory mediator production and activation in macrophages. ELJ may inhibit inflammatory responses via suppressing the activation of NF- $\mathrm{KB}$ and $\mathrm{p} 38$ signaling pathways in LPS-stimulated macrophages. ELJ, ethanol extract of Lygodium japonicum spores; NF, nuclear factor; LPS, lipopolysaccharide; TLR, Toll-like receptor; TAK, transforming growth factor $\beta$-activated kinase; MAP3K8, mitogen-activated protein kinase kinase kinase 8; IKK, inhibitor of $\kappa \mathrm{B}$ kinase; MKK, mitogen-activated protein kinase kinase; I $\mathrm{B} \alpha$, inhibitor of $\kappa \mathrm{B} \alpha$; JNK, c-Jun N-terminal kinase; ERK, extracellular signal-regulated kinase; p-, phosphorylated; NO, nitric oxide; $\mathrm{IL}$, interleukin; TNF, tumor necrosis factor. 
the treatment of severe inflammation $(35,36)$. In the present study, ELJ was demonstrated to attenuate the LPS-stimulated production of proinflammatory mediators in RAW264.7 macrophages in vitro, without exerting cytotoxic effects at low concentrations. These findings suggested that ELJ may have potential as an anti-inflammatory phytomedicine for the treatment of inflammatory disorders.

The results of the present study demonstrated that ELJ suppressed the expression of iNOS and the production of proinflammatory cytokines; however, it failed to inhibit COX-2 expression. The differential inhibitory effects of ELJ on proinflammatory mediators may be attributed to the selective regulation of proinflammatory signal transduction pathways. These findings are in accordance with our previous studies, which have reported that anti-inflammatory extracts, including the ethanol extract of Crataeva nurvala and the methanol extract of Xanthium sibiricum root, selectively regulated major proinflammatory signaling pathways and differentially modulated the production of proinflammatory mediators $(21,37)$. Furthermore, previous studies have reported that the anti-inflammatory properties of several agents, including roscovitine and paeonol, were associated with the selective inhibition of particular inflammatory mediators due to the differential modulation of specific inflammatory signaling pathways $(38,39)$. However, the molecular mechanisms underlying the selective regulatory effects of ELJ on the production of proinflammatory mediators have yet to be elucidated. It may be hypothesized that the components of ELJ exhibit selectivity toward specific inflammatory signaling pathways. Previous studies have demonstrated that natural extracts regulated the production of proinflammatory mediators through the selective inhibition of NF- $\mathrm{B}$ - and MAPK-mediated signaling pathways, due to the different actions of the various bioactive components in the extracts $(40,41)$. Based on the component analysis and bioassay with the extracts of interest, it may be hypothesized that the selective regulation of inflammatory responses may be attributed to the specific effects of each component in the extract. Furthermore, ELJ contains several anti-inflammatory components, including fatty acids and flavonoids (42-44), thus suggesting that the differential effects of ELJ on proinflammatory mediator production and signal transduction may be due to the selective actions of its various components.

ELJ contains fatty acids, flavones, phenolic acids, and phenolic glycosides (17-19). Among them, oleic acid has been reported to inhibit saturated fatty acid-induced inflammatory responses in human aortic endothelial cells through the inhibition of NF- $\kappa \mathrm{B}$ signaling (45). Furthermore, linoleic acid has been revealed to exhibit anti-inflammatory effects, via inhibiting $\mathrm{NF}-\kappa \mathrm{B}$ and MAPK pathways in endothelial cells or Helicobacter pylori-infected gastric epithelial cells in vitro $(46,47)$. The results of the present study demonstrated that NF- $\kappa \mathrm{B}$ and $\mathrm{p} 38$ signal transduction pathways were involved in the anti-inflammatory effects of ELJ, thus suggesting that the anti-inflammatory properties of ELJ may be attributed to the oleic and linoleic acid components of the extract.

In conclusion, in the present study, ELJ was revealed to suppress the production of proinflammatory mediators, including iNOS, IL-1 $\beta$, IL-6 and TNF- $\alpha$, possibly through the inhibition of $\mathrm{NF}-\kappa \mathrm{B}$ and $\mathrm{p} 38$ signaling pathways in
LPS-stimulated macrophages in vitro. Based on the ethnopharmacological use of ELJ for the treatment of inflammatory disorders and the presently demonstrated in vitro anti-inflammatory properties, it may be hypothesized that ELJ has potential as an alternative therapeutic strategy for the treatment of inflammatory conditions.

\section{Acknowledgements}

The present study was supported by the National Research Foundation of Korea funded by the Ministry of Science, ICT \& Future Planning (grant nos. NRF-2015R1A2A2A11001446 and NRF-2015R1A5A1008958) and Ministry of Education, Science and Technology (grant no. NRF-2016R1A6A3A11931134).

\section{References}

1. Fujiwara $\mathrm{N}$ and Kobayashi K: Macrophages in inflammation. Curr Drug Targets Inflamm Allergy 4: 281-286, 2005.

2. Nathan CF: Secretory products of macrophages. J Clin Invest 79: 319-326, 1987.

3. Watkins LR, Maier SF and Goehler LE: Immune activation: The role of pro-inflammatory cytokines in inflammation, illness responses and pathological pain states. Pain 63: 289-302, 1995.

4. McCubrey JA, LaHair MM and Franklin RA: Reactive oxygen species-induced activation of the MAP kinase signaling pathways. Antioxid Redox Signal 8: 1775-1789, 2006.

5. Tak PP and Firestein GS: NF-карраB: A key role in inflammatory diseases. J Clin Invest 107: 7-11, 2001.

6. Mahdavi J, Sondén B, Hurtig M, Olfat FO, Forsberg L, Roche N, Angstrom J, Larsson $\mathrm{T}$, Teneberg $\mathrm{S}$, Karlsson KA, et al: Helicobacter pylori SabA adhesin in persistent infection and chronic inflammation. Science 297: 573-578, 2002.

7. Ohshima H, Tatemichi M and Sawa T: Chemical basis of inflammation-induced carcinogenesis. Arch Biochem Biophys 417: 3-11, 2003

8. Akiyama H, Barger S, Barnum S, Bradt B, Bauer J, Cole GM, Cooper NR, Eikelenboom P, Emmerling M, Fiebich BL, et al: Inflammation and Alzheimer's disease. Neurobiol Aging 21: 383-421, 2000.

9. Hart LA, Krishnan VL, Adcock IM, Barnes PJ and Chung KF: Activation and localization of transcription factor, nuclear factor-карраB, in asthma. Am J Respir Crit Care Med 158: 1585-1592, 1998

10. Mahida YR: The key role of macrophages in the immunopathogenesis of inflammatory bowel disease. Inflamm Bowel Dis 6: 21-33, 2000.

11. Eisenberg A, Amato PJ and Tao D: Kam Guilzhouh nyim Guangxxih di Benxtux Wenchual nyim Zihyuanc dih Gonxliix: Kam local indigenous knowledge and sustainable resource management in Guizhou and Guangxi provinces, China. Ethnobotany Res Applications 7: 67-113, 2009.

12. Li X, Zhou A and Han Y: Anti-oxidation and anti-microorganism activities of purification polysaccharide from Lygodium japonicum in vitro. Carbohydrate Polymers 66: 34-42, 2006.

13. Matsuda H, Yamazaki M, Naruo S, Asanuma Y and Kubo M: Anti-androgenic and hair growth promoting activities of Lygodii spora (spore of Lygodium japonicum) I. Active constituents inhibiting testosterone 5alpha-reductase. Biol Pharm Bull 25: 622-626, 2002.

14. Puri H: Indian pteridophytes used in folk remedies. Am Fern J 60: 137-143, 1970.

15. Cho HJ, Bae WJ, Kim SJ, Hong SH, Lee JY, Hwang TK, Choi YJ, Hwang SY and Kim SW: The inhibitory effect of an ethanol extract of the spores of Lygodium japonicum on ethylene glycol-induced kidney calculi in rats. Urolithiasis 42: 309-315, 2014.

16. Won JB and Ma CJ: Neuroprotective activities of some medicinal plants against glutamate-induced neurotoxicity in primary cultures of rat cortical cells. Natural Product Sci 15: 125-129, 2009.

17. Zhang LH, Yin ZQ, Ye WC, Zhao SX, Wang L and Hu F: Studies on the chemical constituents in herb of Lygodium japonicum. Zhongguo Zhong Yao Za Zhi 30: 1522-1524, 2005 (In Chinese). 
18. Zhang LH, Fan CL, Zhang XT, Yin ZQ and Ye WC: A new steroidal glycoside from Lygodium japonicum. J China Pharmaceutical Univ 37: 491-493, 2006.

19. Zhang LH, Yin ZQ and Ye WC: Flavonoids from Lygodium japonicum. Biochem Systemat Ecol 34: 885-886, 2006.

20. Livak KJ and Schmittgen TD: Analysis of relative gene expression data using real-time quantitative PCR and the 2(-Delta Delta C(T)) method. Methods 25: 402-408, 2001.

21. Cho YC, Ju A, Kim BR and Cho S: Anti-inflammatory effects of Crataeva nurvala Buch. Ham. are mediated via inactivation of ERK but not NF-kB. J Ethnopharmacol 162: 140-147, 2015.

22. Sharma JN, Al-Omran A and Parvathy SS: Role of nitric oxide in inflammatory diseases. Inflammopharmacology 15: 252-259, 2007.

23. Wallace JL: Nitric oxide as a regulator of inflammatory processes. Mem Inst Oswaldo Cruz 100 (Suppl 1): S5-S9, 2005.

24. Kalinski P: Regulation of immune responses by prostaglandin E2. J Immunol 188: 21-28, 2012.

25. Karin M: The regulation of AP-1 activity by mitogen-activated protein kinases. J Biol Chem 270: 16483-16486, 1995.

26. Karin M and Delhase M: The I kappa B kinase (IKK) and NF-kappa B: Key elements of proinflammatory signalling. Semin Immunol 12: 85-98, 2000.

27. Verma IM, Stevenson JK, Schwarz EM, Van Antwerp D and Miyamoto S: Rel/NF-kappa B/I kappa B family: Intimate tales of association and dissociation. Genes Dev 9: 2723-2735, 1995.

28. Adcock IM: Transcription factors as activators of gene transcription: AP-1 and NF-kappa B. Monaldi Arch Chest Dis 52: 178-186, 1997.

29. Weinstein SL, Sanghera JS, Lemke K, DeFranco AL and Pelech SL: Bacterial lipopolysaccharide induces tyrosine phosphorylation and activation of mitogen-activated protein kinases in macrophages. J Biol Chem 267: 14955-14962, 1992.

30. Yamamoto Y, Yin MJ and Gaynor RB: IkappaB kinase alpha (IKKalpha) regulation of IKKbeta kinase activity. Mol Cell Biol 20: 3655-3666, 2000.

31. Yang Y, Kim SC, Yu T, Yi YS, Rhee MH, Sung GH, Yoo BC and Cho JY: Functional roles of p38 mitogen-activated protein kinase in macrophage-mediated inflammatory responses. Mediators Inflamm 2014: 352371, 2014.

32. Connelly L, Palacios-Callender M, Ameixa C, Moncada S and Hobbs AJ: Biphasic regulation of NF-kappa B activity underlies the pro- and anti-inflammatory actions of nitric oxide. J Immunol 166: 3873-3881, 2001.

33. Sweet MJ and Hume DA: Endotoxin signal transduction in macrophages. J Leukoc Biol 60: 8-26, 1996.

34. Asehnoune K, Strassheim D, Mitra S, Kim JY and Abraham E: Involvement of reactive oxygen species in Toll-like receptor 4-dependent activation of NF-kappa B. J Immunol 172: 2522-2529, 2004.

35. Saba E, Jeong DH, Roh SS, Kim SH, Kim SD, Kim HK and Rhee MH: Black ginseng-enriched Chong-Myung-Tang extracts improve spatial learning behavior in rats and elicit anti-inflammatory effects in vitro. J Ginseng Res 41: 151-158, 2017.
36. Ren K, Jin C, Ma P, Ren Q, Jia Z and Zhu D: Ginsenoside Rd alleviates mouse acute renal ischemia/reperfusion injury by modulating macrophage phenotype. J Ginseng Res 40: 196-202, 2016.

37. Ju A, Cho YC and Cho S: Methanol extracts of Xanthium sibiricum roots inhibit inflammatory responses via the inhibition of nuclear factor-kB (NF-kB) and signal transducer and activator of transcription 3 (STAT3) in murine macrophages. J Ethnopharmacol 174: 74-81, 2015.

38. Du J, Wei N, Guan T, Xu H, An J, Pritchard KA Jr and Shi Y: Inhibition of CDKS by roscovitine suppressed LPS-induced *NO production through inhibiting NFkappaB activation and $\mathrm{BH} 4$ biosynthesis in macrophages. Am J Physiol Cell Physiol 297: C742-C749, 2009.

39. Jin X, Wang J, Xia ZM, Shang CH, Chao QL, Liu YR, Fan HY, Chen DQ, Qiu F and Zhao F: Anti-inflammatory and anti-oxidative activities of paeonol and its metabolites through blocking MAPK/ERK/p38 signaling pathway. Inflammation 39: 434-446, 2016.

40. Koo HJ, Yoon WJ, Sohn EH, Ham YM, Jang SA, Kwon JE, Jeong YJ, Kwak JH, Sohn E, Park SY, et al: The analgesic and anti-inflammatory effects of Litsea japonica fruit are mediated via suppression of NF-kB and JNK/p38 MAPK activation. Int Immunopharmacol 22: 84-97, 2014.

41. Qian Z, Wu Z, Huang L, Qiu H, Wang L, Li L, Yao L, Kang K, $\mathrm{Qu} \mathrm{J}, \mathrm{Wu} \mathrm{Y}$, et al: Mulberry fruit prevents LPS-induced NF-kB/pERK/MAPK signals in macrophages and suppresses acute colitis and colorectal tumorigenesis in mice. Sci Rep 5: 17348, 2015.

42. Comalada M, Ballester I, Bailón E, Sierra S, Xaus J, Gálvez J, de Medina FS and Zarzuelo A: Inhibition of pro-inflammatory markers in primary bone marrow-derived mouse macrophages by naturally occurring flavonoids: Analysis of the structure-activity relationship. Biochem Pharmacol 72: 1010-1021, 2006.

43. Park SY, Seetharaman R, Ko MJ, Kim DY, Kim TH, Yoon MK, Kwak JH, Lee SJ, Bae YS and Choi YW: Ethyl linoleate from garlic attenuates lipopolysaccharide-induced pro-inflammatory cytokine production by inducing heme oxygenase-1 in RAW264.7 cells. Int Immunopharmacol 19: 253-261, 2014.

44. Yu Y, Correll PH and Vanden Heuvel JP: Conjugated linoleic acid decreases production of pro-inflammatory products in macrophages: Evidence for a PPAR gamma-dependent mechanism. Biochim Biophys Acta 1581: 89-99, 2002.

45. Harvey KA, Walker CL, Xu Z, Whitley P, Pavlina TM, Hise M, Zaloga GP and Siddiqui RA: Oleic acid inhibits stearic acid-induced inhibition of cell growth and pro-inflammatory responses in human aortic endothelial cells. J Lipid Res 51: 3470-3480, 2010

46. Lee SE, Lim JW, Kim JM and Kim H: Anti-inflammatory mechanism of polyunsaturated fatty acids in Helicobacter pylori-infected gastric epithelial cells. Mediators Inflamm 2014: 128919, 2014

47. Young VM, Toborek M, Yang F, McClain CJ and Hennig B: Effect of linoleic acid on endothelial cell inflammatory mediators. Metabolism 47: 566-572, 1998. 